\title{
FAULT TOLERANT CONTROL DESIGN FOR POLYTOPIC LPV SYSTEMS
}

\author{
Mickael RODRIGUES *, Didier THEILliOL **, SAMir ABERKANE **, \\ DOMINIQUE SAUTER ** \\ ${ }^{*}$ Laboratoire d'Automatique et de Génie des Procédés, LAGEP-UMR-CNRS 5007 \\ Université Claude Bernard Lyon I CPE, Lyon, Bd du 11 Novembre 1918 \\ F-69622 Villeurbanne Cedex, France \\ e-mail: rodrigues@lagep.univ-1yon1.fr \\ ** Centre de Recherche en Automatique de Nancy, CRAN - CNRS - INPL - UHP - UMR 7039 \\ BP 239, F-54506 Vandœuvre-lès-Nancy Cedex, France \\ e-mail: didier.theilliol@cran.uhp-nancy.fr
}

\begin{abstract}
This paper deals with a Fault Tolerant Control (FTC) strategy for polytopic Linear Parameter Varying (LPV) systems. The main contribution consists in the design of a Static Output Feedback (SOF) dedicated to such systems in the presence of multiple actuator faults/failures. The controllers are synthesized through Linear Matrix Inequalities (LMIs) in both faultfree and faulty cases in order to preserve the system closed-loop stability. Hence, this paper provides a new sufficient (but not necessary) condition for the solvability of the stabilizing output feedback control problem. An example illustrates the effectiveness and performances of the proposed FTC method.
\end{abstract}

Keywords: fault tolerant control, multiple actuator failures, polytopic LPV systems, LMI, static output feedback, stability

\section{Introduction}

As performance requirements increase in advanced technological systems, their associated control systems become more and more complex. At the same time, complicated systems could have various consequences in the event of component failures. Therefore, it is very important to consider the safety and fault tolerance of such systems at the design stage. For these safety-critical systems, Fault Tolerant Control Systems (FTCSs) have been developed to meet these essential objectives. FTCSs have been of great practical importance and attracted a lot of interest for the last three decades. Bibliographical reviews on reconfigurable FTCSs can be found in (Patton, 1997; Zhang and Jiang, 2003).

The objective of an FTCS is to maintain current performances close to desirable ones and preserve stability conditions in the presence of component and/or instrument faults; in some circumstances reduced performances could be accepted as a trade-off. In fact, many FTC methods against actuator failures were recently developed in (Blanke et al., 2003; Noura et al., 2000). Almost all methods can be categorized into two groups (Zhang and Jiang, 2003) i.e., passive (Eterno et al., 1985; Veillette, 2002) and active (Theilliol et al., 2002; Wu et $a l .$, 2000; Zhang and Jiang, 2001) approaches.

First of all, passive FTC deals with a presumed set of system component failures based on actuator redundancies at the controller design stage. The resulting controller usually has a fixed structure and parameters. However, the main drawback of passive FTC approaches is that, as the number of potential failures and the degree of system redundancy increase, the controller design could become very complex and the performance of the resulting controller (if it exists) could become significantly conservative. Moreover, if an unanticipated failure occurs, passive FTC cannot ensure system stability and cannot reach again nominal performances. Controller switching underlines the fact that many faulty system representations have to be identified so as to synthesize off-line pre-computed and stabilizing controllers. Furthermore, such identification is sometimes difficult to obtain and it is restrictive to consider only pre-determined actuator faults and not all actuator faults. 
Active FTC strategies make it possible to consider more faults than passive ones do: some research works deal with it and underline the problem of closed-loop system stability in the presence of multiple actuator failures (Kanev, 2004; Maki et al., 2001; Rodrigues et al., 2005a; Theilliol et al., 2003; Wu et al., 2000; Zhang et al., 2005). AFTC is characterized by an on-line Fault Detection and Isolation (FDI) scheme (Rodrigues, 2006) and an automatic control reconfiguration mechanism. Moreover, AFTC is often dedicated to linear systems or the linearization of nonlinear systems, but rarely to Linear Parameter Varying (LPV) systems.

Various system modelling techniques in the faultfree case are presented in (Glover, 2003; Reberga et al., 2005; Wan and Kothare, 2004). They deal with Linear Parameter Varying (LPV) and/or polytopic representations. The main motivation for polytopic LPV or just LPV systems comes from the analysis and control of nonlinear systems. Moreover, to the best of our knowledge, there are few works published on handling multiple actuator failures based on polytopic LPV system representations.

Starting research on FTC and polytopic systems, we can note that multi-models often use polytopic representations. Chadli et al. (2002) developed an output feedback through LMIs in a multi-model context but only in a faultfree case. In (Rodrigues et al., 2005b), a solution was proposed in the same multi-model context with the aim to design a static state feedback which takes into account multiple actuator failures. From a practical point of view, a state feedback needs to use an estimator if not all the states are measurable. It can be difficult to design such state estimators while the system is reconfigured. Therefore, we propose to develop a solution to handle FTC and polytopic LPV systems with an SOF design. An output feedback design is less restrictive than a state feedback design and it can produce solutions to practical FTC problems where only system outputs are available. Output feedback design is also developed in (Geromel et al., 1998) with a sufficient condition for the solvability of the stabilizing SOF control problem and, in (Jabbari, 1997), with structured uncertainty. Also, Rosinova and Vesely (2004) develop a robust SOF for linear discrete-time systems with polytopic uncertainties through an LMI synthesis. However, none of these studies take into account any actuator failures, dealing with linear systems and not with LPV systems.

In this paper, an active FTC strategy is developed to avoid actuator fault/failure effects on polytopic LPV systems. In many research works, feedback design is only used for polytopic LPV systems in the fault-free case (Angelis, 2001; Bouazizi et al., 2001), but does not consider actuator failures. This paper deals with an SOF synthesis in the presence of multiple actuator failures. Under the assumption that a fault is detected, isolated and estimated, the developed method preserves the system perfor- mances through an appropriate controller re-design in the faulty case. Multiple controllers are designed such that any controller can maintain closed-loop stability for any combination of multiple actuator failures.

The paper is organized as follows: Section 2 defines a polytopic LPV system representation under multiple actuator failures. In Section 3, we develop a controller synthesis method for each actuator and generate an output feedback control law for polytopic LPV systems in both the fault-free and faulty cases. The FTC philosophy rests on accurate FDI information. An illustrative example is given in Section 4 to underline the synthesis. Finally, concluding remarks are given in the last section.

\section{Polytopic LPV Systems with Multiple Actuator Failures}

Consider the following discrete LPV representation in the fault-free case:

$$
\begin{aligned}
x_{k+1} & =\widetilde{A}(\theta) x_{k}+\widetilde{B}(\theta) u_{k}, \\
y_{k} & =\widetilde{C}(\theta) x_{k}+\widetilde{D}(\theta) u_{k},
\end{aligned}
$$

where $x \in \mathbb{R}^{n}$ represents the state vector, $u \in \mathbb{R}^{p}$ is the input vector, $y \in \mathbb{R}^{m}$ is the output vector. The system (1) assumes an affine parameter dependence such that $\widetilde{M}(\theta)=\widetilde{M}_{0}+\sum_{j=1}^{v} \theta_{j} \widetilde{M}_{j}$, with the following notation:

$$
\widetilde{M}=\left[\begin{array}{cc}
\widetilde{A} & \widetilde{B} \\
\widetilde{C} & \widetilde{D}
\end{array}\right]
$$

The affine LPV system (1) with bounded parameters $\underline{\theta}_{j} \leq \theta_{j}(k) \leq \bar{\theta}_{j}$ (here $\bar{\theta}_{j}$ and $\underline{\theta}_{j}$ represent the maximum and minimum values of $\theta_{j}$, respectively) can be represented by a polytopic form (Bouazizi et al., 2001; Rodrigues, 2005) when the varying parameter $\theta(k)$ evolves in a polytopic domain $\Theta$ of vertices $\left[\theta_{1}, \theta_{2}, \ldots, \theta_{v}\right]$ (where the vertices are the extreme values of the parameter $\theta$ ). In the following, we consider only strictly proper systems such that $D=0$. The system can be defined via a matrix polytope with summits $S_{j}:=\left[A_{j}, B_{j}, C_{j}\right], \forall j \in[1, \ldots, N]$ and a barycentric combination, where $N=2^{v}$. Consequently, under a multiplicative actuator fault representation (Rodrigues et al., 2005a), the system (1) can be rewritten as the following polytopic representation:

$$
\begin{aligned}
x_{k+1} & =\sum_{j=1}^{N} \alpha_{k}^{j}(\theta)\left[A_{j} x_{k}+B_{j}\left(I_{p}-\gamma\right) u_{k}\right], \\
y_{k} & =\sum_{j=1}^{N} \alpha_{k}^{j}(\theta)\left[C_{j} x_{k}\right],
\end{aligned}
$$

where $\alpha_{k}^{j}(\theta)=\alpha\left(\bar{\theta}_{j}, \underline{\theta}_{j}, \theta_{j}(k), k\right)$ and $\theta_{j}(k)$ is the value of $\theta_{j}$ at the sample $k$, see (Rodrigues, 2005; Da Silva et 
$a l ., 2004)$ for more details about the LPV polytopic representation. Here $A_{j} \in \mathbb{R}^{n \times n}, B_{j} \in \mathbb{R}^{n \times p}, C_{j} \in \mathbb{R}^{m \times n}$ are time-invariant matrices defined for the $j$-th model. The polytopic system is scheduled through functions designed as follows: $\alpha_{k}^{j}(\theta), \forall j \in[1, \ldots, N]$ lie in a convex set

$$
\begin{array}{r}
\Omega=\left\{\alpha_{k}^{j}(\theta) \in \mathbb{R}^{N}, \alpha_{k}(\theta)=\left[\alpha_{k}^{1}(\theta), \ldots, \alpha_{k}^{N}(\theta)\right]^{T},\right. \\
\left.\alpha_{k}^{j}(\theta) \geq 0, \forall j, \sum_{j=1}^{N} \alpha_{k}^{j}(\theta)=1\right\} .
\end{array}
$$

These functions are assumed to be available in real time depending on fault-free parameter measurements (Casavola et al., 2003). The matrix $\gamma$ is defined as follows:

$$
\gamma \triangleq \operatorname{diag}\left[\gamma^{1}, \gamma^{2}, \ldots, \gamma^{p}\right], 0 \leq \gamma^{i} \leq 1
$$

such that for extreme values

$$
\left\{\begin{aligned}
\gamma^{i}=1 \rightarrow & \text { represents a total failure of } \\
& \text { the } i \text {-th actuator, } i \in[1, \ldots, p], \\
\gamma^{i}=0 \rightarrow & \text { denotes the healthy } i \text {-th actuator. }
\end{aligned}\right.
$$

Remark 1. $\gamma^{i}$ can take any value between 0 and 1 . It represents a loss in the effectiveness of the $i$-th actuator, for example, a $70 \%$ loss in the effectiveness of the first actuator will be represented by $\gamma^{1}=0.7$. When an actuator fault appears in the system and the controller is not designed to take account of such a problem, the closedloop system stability cannot be obviously ensured. Consequently, we propose to develop an SOF for polytopic systems with multiple actuator failures.

\section{Fault Tolerant Control Design for Polytopic LPV Systems}

3.1. Nominal Control Law Synthesis. Recall the multiplicative actuator fault representation on a polytopic system as follows:

$$
\begin{aligned}
x_{k+1} & =\sum_{j=1}^{N} \alpha_{k}^{j}\left[A_{j} x_{k}+\sum_{i=1}^{p} B_{j}^{i}\left(I_{p}-\gamma\right) u_{k}\right], \\
y_{k} & =C x_{k},
\end{aligned}
$$

where $\alpha_{k}^{j}$ represents $\alpha_{k}^{j}(\theta)$ for notational simplicity and the matrices $B_{j}^{i}$ represent a total failure in all actuators except the $i$-th one such that

$$
B_{j}^{i}=\left[0, \ldots, 0, b_{j}^{i}, 0, \ldots, 0\right]
$$

and $B_{j}=\left[b_{j}^{1}, b_{j}^{2}, \ldots, b_{j}^{p}\right.$, with $b_{j}^{i} \in \mathbb{R}^{n \times 1}$. Each column of $B_{j}$ is assumed to have full column rank. The following assumptions are made:
Assumption 1. The pairs $\left(A_{j}, b_{j}^{i}\right), \forall i=[1, \ldots, p]$ are assumed to be controllable $\forall j \in[1, \ldots, N]$.

Assumption 2. The matrix $C=C_{j}, \forall j \in[1, \ldots, N]$.

Assumption 3. The matrix $C$ has full row rank.

Assumption 4. At every time instant there is at least one fault-free actuator, which means that the situation $\gamma^{1}=$ $\cdots=\gamma^{p}=1$ is excluded.

In the nominal case, the SOF can be expressed as

$$
u_{k}=-F y_{k},
$$

where $y_{k}=C x_{k}$ and $F \in \mathbb{R}^{p \times m}$ is the output feedback controller gain. In the fault-free case $(\gamma=0)$, the system (5) with a nominal control law $u_{k}=-F y_{k}$ is equivalent to

$$
\begin{aligned}
x_{k+1} & =\sum_{j=1}^{N} \alpha_{k}^{j}\left[A_{j} x_{k}+B_{j}(I-\gamma)\left(-F y_{k}\right)\right] \\
& =\sum_{j=1}^{N} \alpha_{k}^{j}\left(A_{j}-B_{j} F C\right) x_{k} .
\end{aligned}
$$

The stability of the closed-loop system is established using an LMI pole placement technique. For many problems, an exact pole assignment may not be necessary and it suffices to locate the poles of the closed-loop system in a subregion of the complex left half-plane (Chilali and Gahinet, 1996; Rodrigues et al., 2005a).

Consequently, define a disk region LMI $D$ included in the unit circle with an affix $(-q, 0)$ and a radius $r$ such that $(q+r)<1$. These two scalars $q$ and $r$ are used to determine a specific region included in the unit circle so as to place closed-loop system eigenvalues. The pole placement of the closed-loop system (8) for all the models $j \in[1, \ldots, N]$ in the LMI region can be expressed as follows:

$$
\left(\begin{array}{cc}
-r X & q X+\left(A_{j} X-B_{j} F C X\right)^{T} \\
q X+\left(A_{j} X-B_{j} F C X\right) & -r X
\end{array}\right)<0 .
$$

However, these inequalities are no longer linear with respect to the unknown matrices $X=X^{T}>0$ and $F, \forall j \in[1, \ldots, N]$. Therefore, the solution is not guaranteed to belong to a convex domain and the classical tools for solving sets of matrix inequalities cannot be used. This constitutes the major difficulty in output feedback design.

We propose to transform the BMI conditions (9) in $X$ and $F, \forall j \in[1, \ldots, N]$, to LMI conditions which will be used to synthesize directly a stabilizing SOF. We will synthesize the controllers $F_{i}$ for each actuator in order to define an SOF control law.

Theorem 1. Consider the system (5) in the fault-free case $(\gamma=0)$, defined as $\forall j \in[1, \ldots, N]$. Assume that it is possible to find the matrices $X_{i}=X_{i}^{T}>0, M$ and $V_{i} \forall i=$ 
$[1, \ldots, p]$ such that $\forall i=[1, \ldots, p], \forall j=[1, \ldots, N]$ :

$$
\left(\begin{array}{cc}
-r X_{i} & q X_{i}+\left(A_{j} X_{i}-B_{j}^{i} V_{i} C\right)^{T} \\
q X_{i}+A_{j} X_{i}-B_{j}^{i} V_{i} C & -r X_{i}
\end{array}\right)<0
$$

with

$$
C X_{i}=M_{i} C
$$

The control law with the SOF $u_{k}=-F y_{k}$ makes it possible to place the eigenvalues of the closed-loop system (5) in a predetermined LMI-region with $F M=V$,

$$
F=\sum_{i=1}^{p} G_{i} V_{i}\left(C C^{T}\left(C \sum_{i=1}^{p} X_{i} C^{T}\right)^{-1}\right)
$$

or $F=V C C^{T}\left(C X C^{T}\right)^{-1}$, where $G_{i} \in \mathbb{R}^{p \times p}$ is a matrix whose elements are zero except for the diagonal entry $g_{i i}=1$, i.e.

$$
G_{i}=\left[\begin{array}{ccc}
0 & \cdots & 0 \\
\vdots & 1 & \vdots \\
0 & \cdots & 0
\end{array}\right]
$$

Proof. As was proposed in (Rodrigues et al., 2005a), the summation of (10) over the set of actuator indices $i \in$ $[1, \ldots, p]$ of the system (5) for the model $j$ gives

$\sum_{i=1}^{p}\left(\begin{array}{cc}-r X_{i} & q X_{i}+\left(A_{j} X_{i}-B_{j}^{i} V_{i} C\right)^{T} \\ q X_{i}+A_{j} X_{i}-B_{j}^{i} V_{i} C & -r X_{i}\end{array}\right)<0$.

Write $X=\sum_{i=1}^{p} X_{i}$ (with $X=X^{T}>0$ ) to obtain

$$
\left(\begin{array}{cc}
-r X & q X+\left(A_{j} X-\sum_{i=1}^{p} B_{j}^{i} V_{i} C\right)^{T} \\
q X+\left(A_{j} X-\sum_{i=1}^{p} B_{j}^{i} V_{i} C\right) & -r X
\end{array}\right)
$$$$
<0
$$

$\forall i=[1, \ldots, p], \forall j=[1, \ldots, N]$. Now, denote by $V_{i}^{l}$ the $l$-th row of the matrix $V_{i}, i=[1, \ldots, p]$, and $l=1, \ldots, p$, which can be calculated from

$$
V_{i}^{l}=G_{l} V_{i}
$$

Therefore,

$$
\begin{aligned}
\sum_{i=1}^{p} B_{j}^{i} V_{i} C & =\sum_{i=1}^{p}\left[0, \ldots, 0, b_{j}^{i}, 0, \ldots, 0\right] V_{i}^{i} C \\
& =B_{j} \sum_{i=1}^{p} V_{i}^{i} C=B_{j} \sum_{i=1}^{p} G_{i} V_{i} C \\
& =B_{j} V C
\end{aligned}
$$

with $V=\sum_{i=1}^{p} G_{i} V_{i}$.

Moreover, $\forall i=[1, \ldots, p], \forall j=[1, \ldots, N]$ we get

$$
\left(\begin{array}{cc}
-r X & q X+\left(A_{j} X-B_{j} V C\right)^{T} \\
q X+\left(A_{j} X-B_{j} V C\right) & -r X
\end{array}\right)<0 .
$$

The substitution of $V=F M$ and $C X=M C$ in the LMI (16) leads to

$$
\left(\begin{array}{cc}
-r X & q X+\left(A_{j} X-B_{j} F C X\right)^{T} \\
q X+\left(A_{j} X-B_{j} F C X\right) & -r X
\end{array}\right)<0,
$$

$\forall i=[1, \ldots, p], \forall j=[1, \ldots, N]$. We should note that the inequalities (17) are BMIs which cannot be solved with classical tools, but recall the definition of the LMI disk region for the unit circle (9). Multiplying each LMI (16) by $\alpha_{k}^{j}$ and summing the results, we obtain

$$
\left(\begin{array}{cc}
-r X & q X+\sum_{j=1}^{N} \alpha_{k}^{j}\left(A_{j} X-B_{j} V C\right)^{T} \\
q X+\sum_{j=1}^{N} \alpha_{k}^{j}\left(A_{j} X-B_{j} V C\right) & -r X
\end{array}\right)
$$

which is equivalent to

$$
\left(\begin{array}{cc}
-r X & q X+(A(\alpha) X-B(\alpha) V C)^{T} \\
q X+(A(\alpha) X-B(\alpha) V C) & -r X
\end{array}\right)
$$

with $A(\alpha)=\sum_{j=1}^{N} \alpha_{k}^{j} A_{j}$ and $B(\alpha)=\sum_{j=1}^{N} \alpha_{k}^{j} B_{j}$. Since the matrix $C$ is supposed to have full row rank, from (11) we deduce that there exists a non-singular matrix $M=C X C^{T}\left(C C^{T}\right)^{-1}$ and then

$$
F=V M^{-1}=\sum_{i=1}^{p} G_{i} V_{i}\left(C C^{T}\left(C \sum_{i=1}^{p} X_{i} C^{T}\right)^{-1}\right) .
$$

Accordingly, the quadratic $\mathcal{D}$-stability is ensured by solving (18) with the SOF $u_{k}=-F y_{k}$.

In the nominal case, we do not really need Assumption 1 in the sense that the proposed SOF is sufficient by solving the LMI (10) with (11). However, in the faulty case, as the proposed FTC method considers actuators which are out of order, we have to assume that each pair $\left(A_{j}, b_{j}^{i}\right)$ is controllable because the loss of one actuator can make the system unstable if Assumption 1 is not considered. Moreover, if Assumption 1 is not satisfied, attempts to find a solution to (10) and (11) will be pointless since the pole placement is obviously impossible for each separate controller. 
3.2. Principles of the Fault Tolerant Control Strategy. The AFTC strategy presented in this paper is able to design a reconfigured controller from the nominal one with an exact fault estimation coming from the FDI scheme, i.e., $\hat{\gamma}=\gamma$. With no loss of generality, the matrix $\gamma$ in (5) is assumed to be decomposed as follows:

$$
\gamma=\left[\begin{array}{cc}
\gamma_{p-h} & 0 \\
0 & I_{h}
\end{array}\right]
$$

Thus, $\gamma$ is a diagonal matrix such that $\gamma_{p-h}$ constitutes a diagonal matrix whose elements $\gamma_{p-h}^{i}, i \in[1, \ldots, p]$ are different from 1 , which represents the number of actuators which are not out of order $\left(\gamma^{i} \neq 1\right)$, and $I_{h}$ represents the number $h$ of totally failed actuators. By recalling $\gamma$ in (20), define $\Gamma$ such that

$$
\begin{aligned}
\Gamma & \triangleq\left[\begin{array}{cc}
I_{p-h}-\gamma_{p-h} & 0 \\
0 & 0_{h}
\end{array}\right]\left[\begin{array}{cc}
\left(I_{p-h}-\gamma_{p-h}\right)^{-1} & 0 \\
0 & 0_{h}
\end{array}\right] \\
& =\left[\begin{array}{cc}
I_{p-h} & 0 \\
0 & O_{h}
\end{array}\right]
\end{aligned}
$$

where $0_{h}$ represents actuators which are out of order and $I_{p-h}$ represents governable ones. The corresponding matrix decomposition of $B$ is

$$
B=\left[\begin{array}{ll}
B_{p-h} & B_{h}
\end{array}\right],
$$

where $B_{p-h} \in \mathbb{R}^{n \times(p-h)}$ and $B_{h} \in \mathbb{R}^{n \times h}$. We will present a control law able to suppress actuator faults in the state space representation (3) and to ensure closedloop stability despite multiple actuator failures. Based on a multiplicative fault representation (5), we propose to use the following control law $u_{\mathrm{FTC}}$ that must suppress all actuator faults in the system (5):

$$
\begin{aligned}
u_{\mathrm{FTC}}= & {\left[\begin{array}{cc}
\left(I_{p-h}-\gamma_{p-h}\right)^{-1} & 0 \\
0 & 0_{h}
\end{array}\right] u_{\mathrm{nom}} } \\
= & {\left[\begin{array}{c}
I_{p-h} \\
0_{h \times(p-h)}
\end{array}\right]\left[I_{p-h}-\gamma_{p-h}\right]^{-1} } \\
& \times\left[\begin{array}{ll}
I_{p-h} & 0_{(p-h) \times h}
\end{array}\right] u_{\mathrm{nom}} .
\end{aligned}
$$

Introduce the set of indices of all actuators that are not out of order (Rodrigues, 2005), i.e.,

$$
\Phi \triangleq\left\{i: i \in(1, \ldots, p), \gamma^{i} \neq 1\right\}
$$

and note that

$$
\begin{aligned}
u_{\mathrm{FTC}} & =\left[\begin{array}{cc}
\left(I_{p-h}-\gamma_{p-h}\right)^{-1} & 0 \\
0 & 0_{h}
\end{array}\right] u_{\mathrm{nom}} \\
& =-\left[\begin{array}{cc}
\left(I_{p-h}-\gamma_{p-h}\right)^{-1} & 0 \\
0 & 0_{h}
\end{array}\right] F_{\mathrm{nom}} y_{k} \\
& =-F_{\mathrm{FTC}} y_{k},
\end{aligned}
$$

where $F_{\text {nom }}$ is a nominal controller and $F_{\mathrm{FTC}}$ stands for the new controller gain. Consequently, this specific control law in the state space representation (5) leads to

$$
\begin{aligned}
B_{j}(I-\gamma) u_{\mathrm{FTC}}= & B_{j}\left[\begin{array}{cc}
I_{p-h}-\gamma_{p-h} & 0 \\
0 & 0_{h}
\end{array}\right] \\
& \times\left[\begin{array}{cc}
\left(I_{p-h}-\gamma_{p-h}\right)^{-1} & 0 \\
0 & 0_{h}
\end{array}\right] u_{\mathrm{nom}} \\
& =B_{j} \Gamma u_{\mathrm{nom}}=\sum_{i \in \Phi} B_{j}^{i} u_{\mathrm{nom}}^{i}
\end{aligned}
$$

which avoids the actuator fault effect and where $\sum_{i \in \Phi} B_{j}^{i}$ represents the actuators that are not out of order, i.e., $\sum_{i \in \Phi} B_{j}^{i}=B_{p-h}$, and $u_{\text {nom }}^{i}$ signifies the $i$-th element of $u_{\text {nom }}$. From Assumption 1, due to the fact that each pair $\left(A_{j}, b_{j}^{i}\right), \forall i=[1, \ldots, p]$ is assumed to be controllable $\forall j=[1, \ldots, N]$, the system still remains controllable in spite of actuator failures.

Remark 2. For simplicity, we have assumed that the matrix $\gamma$ can be decomposed as in (20) in order to consider two different cases, which are $\gamma^{i}=1$ for actuators that are out of order and $\gamma^{i} \neq 1$ for actuators that are still in the normal state: it is directly indicated by the FDI scheme. Of course, it is not the only case that the former actuators are always valid and the latter ones are not: Assumption 4 indicates that any actuator can fail but at least one is still governable. Generalizing, recall that each element $\gamma^{i}, i \in[1, \ldots, p]$ (of the diagonal matrix $\gamma$ ) can take any value in $[0, \ldots, 1]$ and write

$$
u_{\mathrm{FTC}}=\left[\begin{array}{c}
u_{\mathrm{FTC}}^{1} \\
\vdots \\
u_{\mathrm{FTC}}^{p}
\end{array}\right] .
$$

Then each element $u_{\text {FTC }}^{i}$ of $u_{\mathrm{FTC}}$ can be calculated as follows:

$$
\begin{array}{ll}
\text { If } \gamma^{i} \neq 1 \text { then } & u_{\mathrm{FTC}}^{i}=\left(1-\gamma^{i}\right)^{-1} u_{\mathrm{nom}}^{i}, \\
\text { If } \gamma^{i}=1 \text { then } & u_{\mathrm{FTC}}^{i}=0 .
\end{array}
$$

Consequently, for (26) and (27), irrespective of the values of $\gamma^{i}, i \in[1, \ldots, p]$, the expression $B_{j}(I-$ $\gamma) u_{\mathrm{FTC}}=\sum_{i \in \Phi} B_{j}^{i} u_{\text {nom }}^{i}$ remains unchanged (as (25)) and the system still remains controllable under Assumption 1. With no loss of generality, in what follows we will consider the case with $\gamma$ defined in (20).

3.3. Synthesis of a Faulty Control Law. Based on the control law of Section 3.1, an FTC method will be 
developed for the system (5) under the assumption that an actuator fault estimate $\widehat{\gamma}$ is exactly known, i.e., $\widehat{\gamma}=\gamma$.

Theorem 2. Consider the system (5) with multiple actuator failures $\left(\gamma^{i} \neq 0\right.$ ) under Assumption $4 \forall j, j=$ $[1, \ldots, N]$ and the set of indices of the actuators which are not out of order (24). Let the matrices $M, X_{i}$ and $V_{i}$ be determined as in Theorem 1 . Then the control law

$$
\begin{aligned}
u_{\mathrm{FTC}}= & -\left[\begin{array}{cc}
\left(I_{p-h}-\gamma_{p-h}\right)^{-1} & 0 \\
0 & 0_{h}
\end{array}\right] \\
& \times\left(\sum_{i \in \Phi} G_{i} V_{i}\left(C C^{T}\left(C \sum_{i \in \Phi} X_{i} C^{T}\right)^{-1}\right)\right) y_{k} \\
= & -\left[\begin{array}{cc}
\left(I_{p-h}-\gamma_{p-h}\right)^{-1} & 0 \\
0 & 0_{h}
\end{array}\right] F_{\mathrm{rec}} y_{k} \\
= & -F_{\mathrm{FTC}} y_{k}
\end{aligned}
$$

with $G_{i} \in \mathbb{R}^{p \times p}$ (a matrix whose elements are zero except for the diagonal entry $g_{i i}=1$ ) stabilizes the closed-loop system and places the closed-loop poles in the following LMI stability region:

$$
\begin{gathered}
\left(\begin{array}{cc}
-r X & q X+\left(A_{j} X-B_{j} F_{\text {rec }} C X\right)^{T} \\
q X+\left(A_{j} X-B_{j} F_{\text {rec }} C X\right) & -r X \\
<0
\end{array}\right.
\end{gathered}
$$

The SOF control law $u_{k}=-F_{\mathrm{FTC}} y_{k}$ is computed with $F_{\text {rec }} M=V$, where

$$
\begin{aligned}
F_{\text {rec }} & =\sum_{i \in \Phi} G_{i} V_{i}\left(C C^{T}\left(C \sum_{i \in \Phi} X_{i} C^{T}\right)^{-1}\right. \\
& =V C C^{T}\left(C X C^{T}\right)^{-1} .
\end{aligned}
$$

Proof. Applying the new control law (28) to the faulty system (5) leads to the following equation:

$$
\begin{aligned}
B_{j} & (I-\gamma) u_{\mathrm{FTC}} \\
& =-B_{j} \Gamma\left(\sum_{i \in \Phi} G_{i} V_{i}\left(C C^{T}\left(C \sum_{i \in \Phi} X_{i} C^{T}\right)^{-1}\right)\right) y_{k}
\end{aligned}
$$

with $\Gamma$ calculated in (21) and defined as

$$
\Gamma=\left[\begin{array}{cc}
I_{p-h} & 0 \\
0 & O_{h}
\end{array}\right] .
$$

Here $\Gamma$ is a diagonal matrix that contains only entries which are zero (they represent total faults) or one (no fault), cf. Section 3.2. Since $B_{j} \Gamma=$ $\sum_{i \in \Phi} B_{i}^{j}$ characterizes only the actuators which are not out of order, performing the summations in the proof of Theorem 1 over the elements of $\Phi$ shows that $\sum_{i \in \Phi} G_{i} V_{i}\left(C C^{T}\left(C \sum_{i \in \Phi} X_{i} C^{T}\right)^{-1}\right)$ is the output feedback gain matrix for the faulty system $\left(A_{j}, \sum_{i \in \Phi} B_{i}^{j}, C\right)$.
The pairs $\left(A_{j}, b_{j}^{i}\right), \forall i=[1, \ldots, p]$ are assumed to be controllable $\forall j=[1, \ldots, N]$ because we consider the case of actuators which are out of order: the system has to be controllable with at least one actuator. Moreover, if there is a solution for each LMI in (10) and (11), this means that each pair $\left(A_{j}, b_{j}^{i}\right)$ is controllable. However, Assumption 1 does not guarantee the feasibility of (10) and (11), i.e., the proposed SOF solution is only sufficient and not necessary for computing the controller.

\section{Illustrative Example}

The feature of the proposed scheme and the effectiveness of the fault-tolerant control system are developed using an illustrative example with an SOF for a polytopic LPV system. We present the case of two actuator faults which make the closed-loop system unstable. Consider a system described by $N=4$ unstable models. These four models can be adapted from an LPV model, where each of them represents a vertex, as is done in (Glover, 2003) or in (Da Silva et al., 2004), where an aluminum cantilever beam is considered under parametric uncertainties. The discrete state space representation (5) consists of the following matrices:

$$
\begin{aligned}
A_{1}= & {\left[\begin{array}{cccc}
0.75 & 0 & 0 & 0 \\
0 & 0.85 & 0 & 0 \\
0 & 0 & 1.25 & 0 \\
0 & 0 & 0 & 1.5
\end{array}\right], } \\
A_{4}= & {\left[\begin{array}{cccc}
0.6375 & 0 & 0 & 0 \\
0 & 0.7225 & 0 & 0 \\
0 & 0 & 1.0625 & 0 \\
0 & 0 & 0 & 1.275
\end{array}\right], } \\
A_{3}= & {\left[\begin{array}{cccc}
0.525 & 0 & 0 & 0 \\
0 & 0.595 & 0 & 0 \\
0 & 0 & 0.875 & 0 \\
0 & 0 & 0 & 1.05
\end{array}\right], } \\
A_{2}= & {\left[\begin{array}{cccc}
0.6 & 0 & 0 & 0 \\
0 & 0.68 & 0 & 0 \\
0 & 0 & 1 & 0 \\
0 & 0 & 0 & 1.2
\end{array}\right], } \\
C= & {\left[\begin{array}{llll}
0 & 0 & 0 \\
0 & 0 & 1 & 0 \\
0 & 0 & 0 & 1
\end{array}\right], \quad B_{1}=\left[\begin{array}{ll}
1 \\
1 \\
1 \\
1 & 1 \\
1 & 1
\end{array}\right] . }
\end{aligned}
$$

The other matrices are $B_{2}=0.8 B_{1}, B_{3}=0.7 B_{1}$ and $B_{4}=0.85 B_{1}$. The system is in closed loop with the SOF

$$
u_{k}=-\left[\begin{array}{cc}
\left(I_{p-h}-\gamma_{p-h}\right)^{-1} & 0 \\
0 & 0_{h}
\end{array}\right] F y_{k}
$$


(with $y_{k}=C x_{k}$ ), which is synthesized using Theorems 1 and 2. The following matrices are produced directly from Theorem 1 (with Tklmitool version 2.2, which is a Matlab-based graphical user interface to semidefinite programming (SeDuMi) developed by R. Nikoukhah, F. Delebecque, J.-L. Commeau and L. El Ghaoui, and later upgraded by L. Paolopoli, see http://www. eecs . berkeley.edu/ elghaoui/links.htm) with the parameters $q=-0.05, r=0.93$ arbitrarily chosen for stabilizing the closed-loop system:

$$
\begin{aligned}
V_{1}= & {\left[\begin{array}{ccc}
-0.157 & -0.153 & -0.132 \\
0 & 0 & 0
\end{array}\right], } \\
V_{2}= & {\left[\begin{array}{ccc}
0 & 0 & 0 \\
-0.157 & -0.153 & -0.132
\end{array}\right], } \\
X_{1}= & {\left[\begin{array}{cccc}
1 & 0 & 0 & 0 \\
0 & 0.9680 & 0.1074 & 0.1079 \\
0 & 0.1074 & 0.1738 & 0.1341 \\
0 & 0.1079 & 0.1341 & 0.1071
\end{array}\right], } \\
M_{1}= & {\left[\begin{array}{cccc}
0.9680 & 0.1074 & 0.1079 \\
0.1074 & 0.1738 & 0.1341 \\
0.1079 & 0.1341 & 0.1071
\end{array}\right], }
\end{aligned}
$$

with $X_{1}=X_{2}, M_{1}=M_{2}$ and

$$
\begin{aligned}
F & =V M^{-1}=\sum_{i=1}^{p} G_{i} V_{i}\left(C C^{T}\left(C \sum_{i=1}^{p} X_{i} C^{T}\right)^{-1}\right) \\
& =\left[\begin{array}{rrr}
-0.0253 & -1.2221 & 2.1734 \\
-0.0253 & -1.2221 & 2.1734
\end{array}\right], \\
G_{1} & =\left[\begin{array}{ll}
1 & 0 \\
0 & 0
\end{array}\right], \quad G_{2}=\left[\begin{array}{ll}
0 & 0 \\
0 & 1
\end{array}\right] .
\end{aligned}
$$

The parameters $q$ and $r$ were chosen taking account the system eigenvalues in the complex plane without the FTC strategy. An LMI-region is defined as the unit circle (see Section 3.1) with an affix $(-q, 0)$ and a radius $r$. For the same example we can define different combinations of parameters, i.e., different LMI-regions. This LMI-region allows us to place the system eigenvalues in a stable region in spite of actuator failures: it is represented in Fig. 5 with a dashed circle.

Figure 1 represents the parameter evolution in the nominal case: the system outputs (a), the second actuator (b), the first actuator (c) and the parameter evolution $\alpha_{k}^{j}$ (d). The closed-loop system is stable without any fault. At the sample $k=2$, the first actuator is out of order and also an actuator fault with a $60 \%$ loss in effectiveness appears on the second actuator. The matrix $\gamma$ is equal to

$$
\gamma=\left[\begin{array}{cc}
1 & 0 \\
0 & 0.6
\end{array}\right], \quad k \geq 2
$$

Figure 2 represents the outputs in different situations: (a) the nominal case, $(b)$ the faulty case with a failure of the first actuator and a fault in the second actuator at the sample $k=2$ and, finally, $(c)$ the reconfiguration case at the time instant $k=15 \mathrm{~s}$. Figure 2(b) illustrates the instability of the closed-loop system in the faulty case and Figure 2(c) illustrates the contribution of the proposed fault tolerant control: the outputs converge toward their nominal values.

Moreover, the corresponding actuator signals are depicted in Figs. 3 and 4. Figures 3(a) and 4(a) correspond to the actuators in the nominal case and Fig. 3(b) illustrates the loss of the first actuator. Figure 4(b) illustrates the instability of the second actuator in the faulty case and Fig. 4 (c) the reconfigured control law with the second actuator.

In order to simulate a time delay of the FDI block, the new control law is only applied at the sample $k=15$, see Figs. 2(c) and 4(c). Shin (2003) discusses issues with a time delay in an FTC reconfiguration. The reader could refer to this report for more information on time delay in reconfiguration. We do not deal more with this issue because we assume that a perfect FDI scheme is available. We observe that the outputs and the control laws converge to zero.

The system is stabilized with the fault tolerant control law in spite of these actuator faults and failures. Figure 5 represents the evolution of closed-loop system eigenvalues which still remain in the unit circle both in the fault-free case (marked with open circles) and the faulty case (marked with asterisks) with the FTC strategy. The LMI-region is represented by a dashed line. Figure 6 represents the evolution of closed-loop system eigenvalues in the faulty case without FTC: we can see that the closedloop system is unstable. Accordingly, the developed FTC strategy allows the system to continue to operate safely in spite of actuator failures.

\section{Conclusion}

The FTC method presented in this paper illustrates the importance of fault tolerant control for polytopic LPV systems. Controllers are designed for each separate actuator through an LMI pole placement in fault-free and faulty cases. The system continues to operate safely and ensures closed-loop stability in spite of the presence of actuator failures. The main contribution is the design of a static output feedback that takes into account the information provided by an FDI scheme. The proposed SOF solution is sufficient and places the eigenvalues of the closed-loop system in a predetermined LMI region inside the unit circle. From the point of view of investigating a new algorithm in FTC, it can constitute a first step to develop a more practical active FTC for nonlinear systems based on a polytopic LPV representation. An example of a poly- 

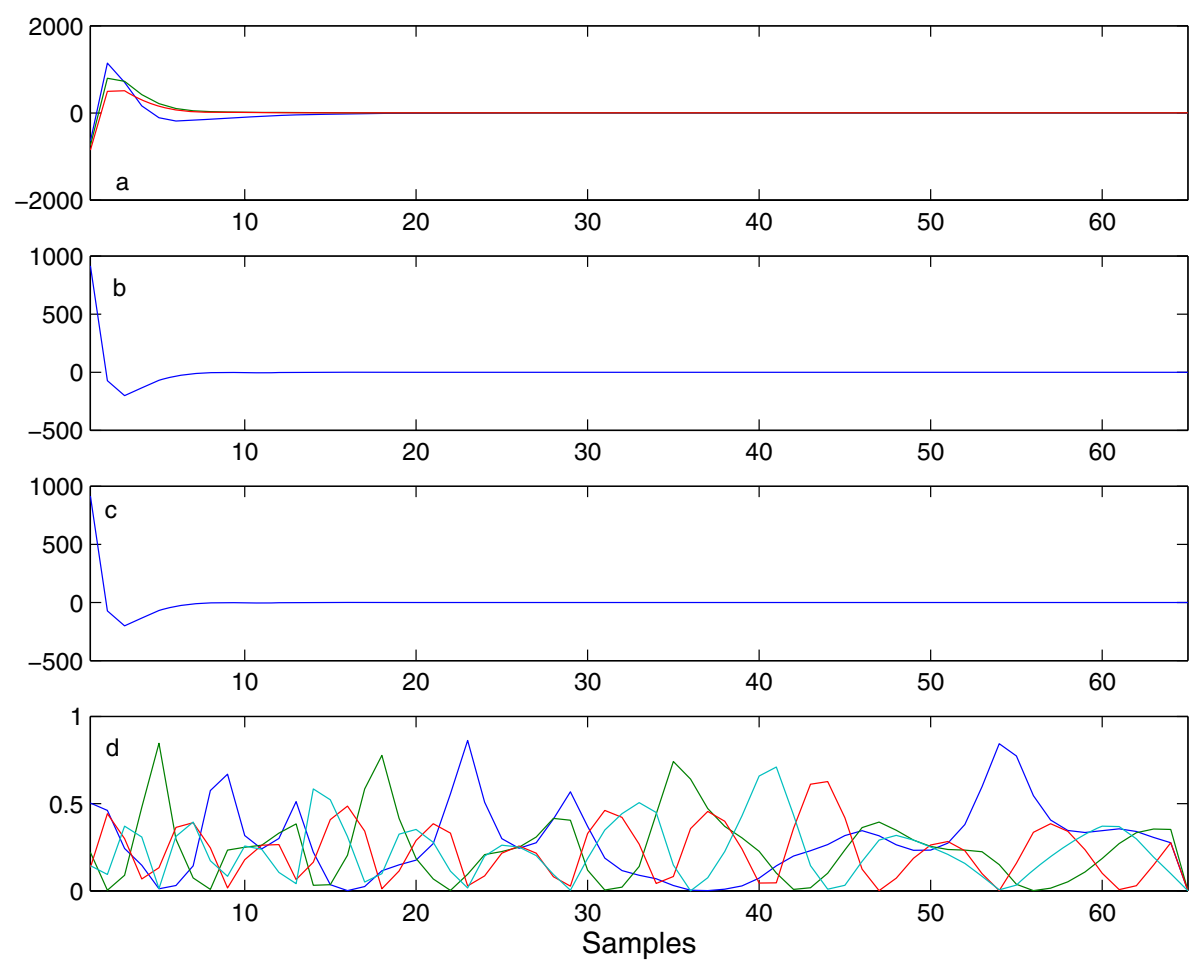

Fig. 1. Nominal case: (a) system outputs, (b) second actuator, (c) first actuator and (d) evolution of the parameter $\alpha_{k}^{j}$.
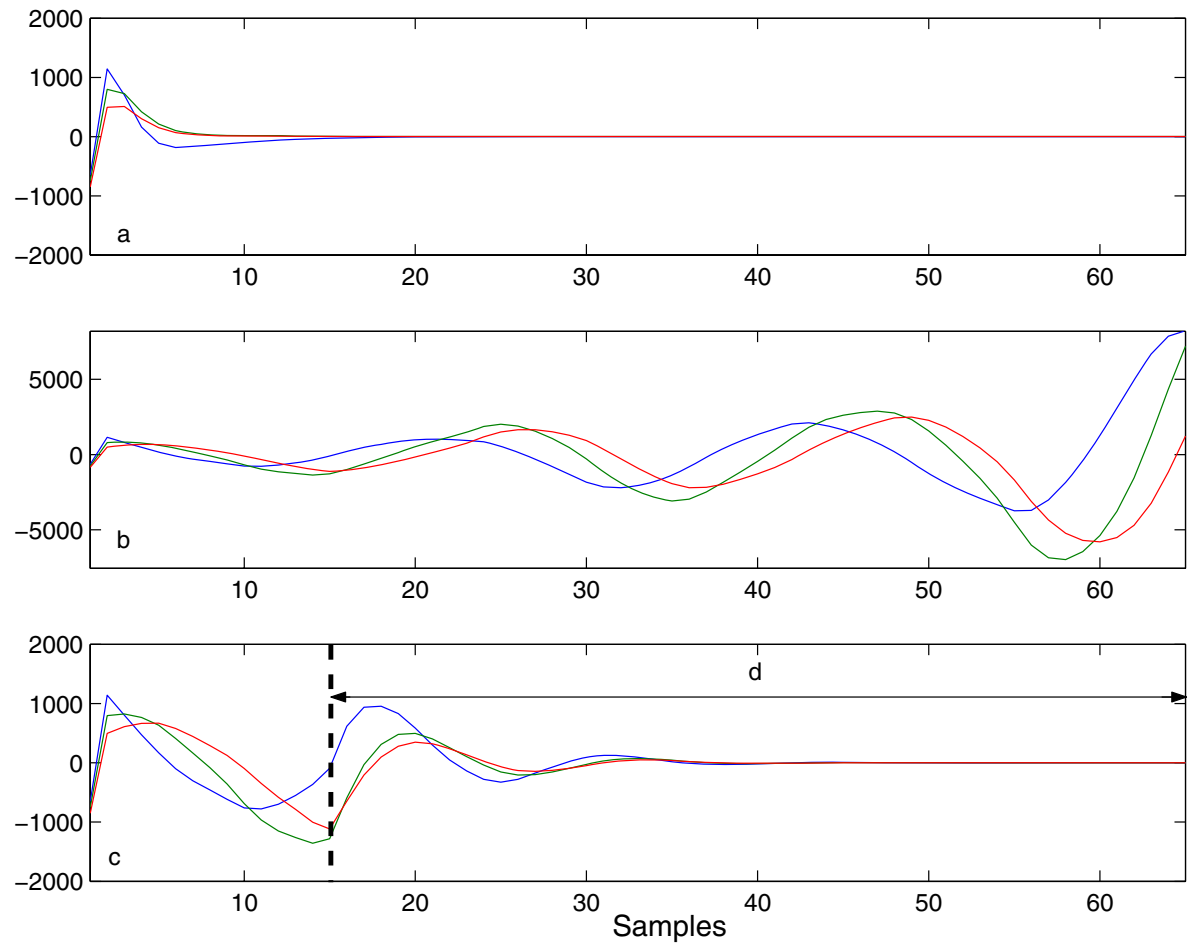

Fig. 2. Outputs: (a) nominal case, (b) faulty case, (c) reconfiguration case. 

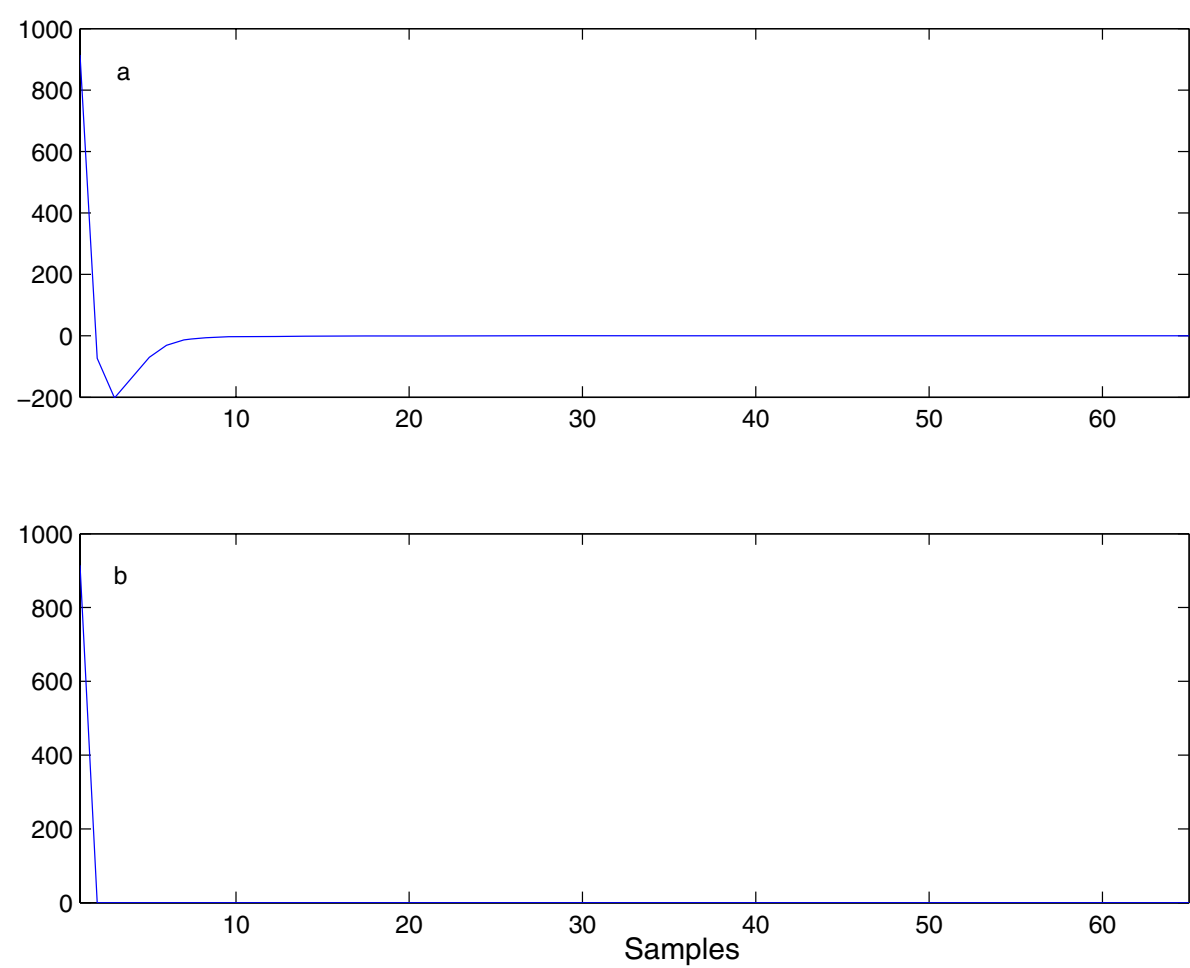

Fig. 3. First actuator: (a) nominal case, (b) faulty and reconfiguration cases.
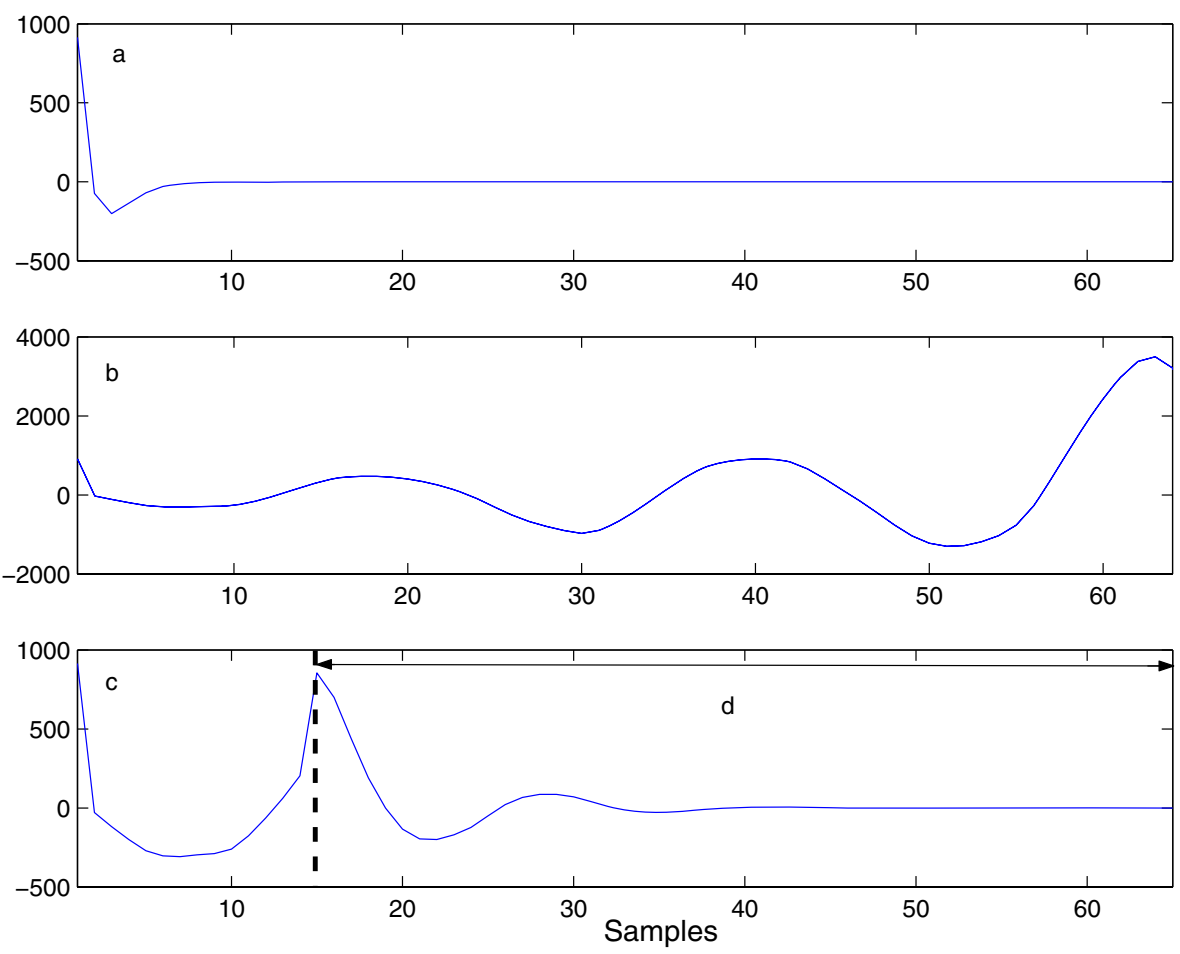

Fig. 4. Second actuator: (a) nominal case, (b) faulty case, (c) reconfiguration case. 


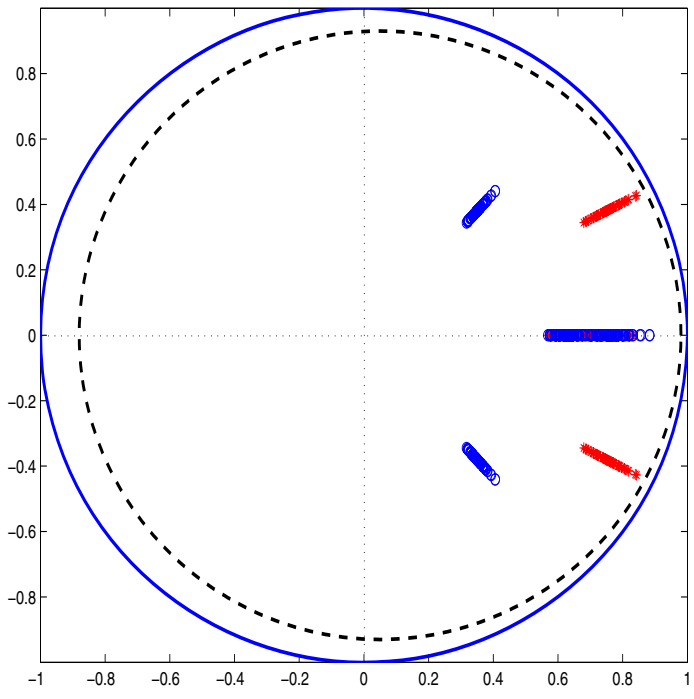

Fig. 5. Domain of the closed-loop system eigenvalues in the fault-free case (marked with open circles) and with the FTC strategy (marked with asterisks).

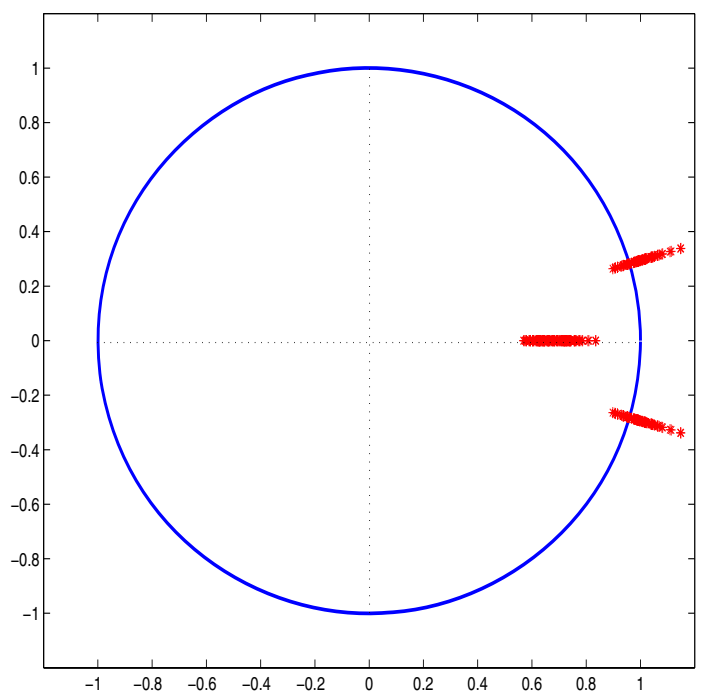

Fig. 6. Domain of the closed-loop system eigenvalues in the faulty case without FTC

topic LPV system was presented to illustrate the effectiveness of the scheme.

\section{Acknowledgments}

We are grateful to the anonymous referees for their constructive comments that have helped us to improve the paper.

\section{References}

Angelis G. Z. (2001): System Analysis, Modelling and Control with Polytopic Linear Models. — Ph.D. thesis, University of Eindhoven, the Netherlands.
Blanke M., Kinnaert M., Lunze J. and Staroswiecki M. (2003): Diagnosis and Fault-Tolerant Control. - Berlin: Springer.

Bouazizi M.H., Kochbati A. and Ksouri M. (2001): $H_{\infty}$ control of LPV systems with dynamic output feedback. - Proc. 9th Mediterranean Conf. Control and Automation (MED'01), Dubrovnik, Croatia, (CD-ROOM).

Casavola A., Famularo D. and Franzč G. (2003): Predictive control of constrained nonlinear systems via LPV linear embeddings. — Int. J. Robust Nonlin. Contr., Vol. 13, Nos. 34, pp. 281-294.

Chadli M., Maquin D., and Ragot J. (2002): An LMI formulation for output feedback stabilization in multiple model approach. - Proc. 41-st IEEE Conf. Decision and Control, Las Vegas, USA, pp. 311-316.

Chilali M. and Gahinet P. (1996): $H_{\infty}$ design with pole placement constraints: An LMI approach. - IEEE Trans. Automat. Contr., Vol. 41, No. 3, pp. 358-367.

Eterno J.S., Looze D.P., Weiss J.L. and Willsky A.S. (1985): Design issues for fault-tolerant restructurable aircraft control. - Proc. 24th IEEE Conf. Decision and Control, Fort Lauderdale, USA, pp. 900-905.

Geromel J.C., DeSouza C.C. and Skelton R.E. (1998): Static output feedback controllers: Stability and convexity. IEEE Trans. Automat. Contr., Vol. 43, No. 1, pp. 120-125.

Glover S.F. (2003): Modeling and Stability Analysis of Power Electronics Based Systems. - Ph.D. thesis, Purdue University, USA.

Jabbari F. (1997): Output feedback controllers for systems with structured uncertainty. - IEEE Trans. Automat. Contr., Vol. 42, No. 5, pp. 715-719.

Kanev S. (2004): Robust Fault-Tolerant Control. — Ph.D. thesis, University of Twente, the Netherlands.

Maki M., Jiang J. and Hagino K. (2001): A stability guaranteed active fault-tolerant control system against actuator failures. - Proc. 40th IEEE Conf. Decision and Control, Orlando, FL, Vol. 2, pp. 1893-1898.

Noura H., Sauter D., Hamelin F. and Theilliol D. (2000): Faulttolerant control in dynamic systems: Application to a winding machine. - IEEE Contr. Syst. Mag., Vol. 20, No. 1, pp. 33-49.

Patton R.J. (1997): Fault-tolerant control: The 1997 situation. - Proc. IFAC Symp. Safeprocess, Kingston Upon Hull, U.K, Vol. 2, pp. 1033-1055.

Reberga L., Henrion D., Bernussou J. and Vary F. (2005): LPV modeling of a turbofan engine. - Proc. 16th IFAC World Congress, Prague, Czech Republic, (CD-ROOM).

Rodrigues M. (2005): Diagnostic et commande active tolérante aux défauts appliqués aux systémes décrits par des multimodčles linéaires. - Ph.D. thesis, Centre de Recherche en Automatique de Nancy, UHP, Nancy, France.

Rodrigues M., Theilliol D., Adam-Medina M. and Sauter D. (2006): A fault detection and isolation scheme for industrial systems based on multiple operating models. - Contr. Eng. Pract. 
Rodrigues M., Theilliol D. and Sauter D. (2005a): Design of an active fault tolerant control and polytopic unknown input observer for systems described by a multi-model representation. - Proc. 44th IEEE Conf. Decision and Control and European Control Conference ECC, Seville, Spain, (CDROM).

Rodrigues M., Theilliol D. and Sauter D. (2005b): Fault tolerant control design of nonlinear systems using LMI gain synthesis. - Proc. 16th IFAC World Congress, Prague, Czech Republic, (CD-ROM).

Rosinova D. and Vesely V. (2004): Robust static output feedback for discrete time systems LMI approach. - Periodica Polytechnica, Vol. 48, No. 3-4, pp. 151-163.

Shin J-Y. (2003): Parameter transient behavior analysis on fault tolerant control system. - Tech. Rep. NASA-CR-2003212682-NIA, Report No. 2003-05, National Institute of Aerospace, Hampton, VA, USA.

Da Silva S., Lopes Junior V. and Assuncao E. (2004): Robust control to parametric uncertainties in smart structures using linear matrix inequalities. - J. Braz. Soc. Mech. Sci. Eng., Vol. 26, No. 4, pp. 430-437.

Theilliol D., Noura H. and Ponsart J.C. (2002): Fault diagnosis and accommodation of three-tank system bsaed on analytical redundancy. - ISA Trans., Vol. 41, No. 3, pp. 365382.
Theilliol D., Sauter D. and Ponsart J.C. (2003): A multiple model Based approach for Fault Tolerant Control in nonlinear systems. - Proc. IFAC Symp. Safeprocess, Washington D.C., (CD-ROM).

Veillette R. (2002): Design of reliable control systems. - IEEE Trans. Automat. Contr., Vol. 37, pp. 290-304.

Wan Z. and Kothare M.V. (2004): Efficient scheduled stabilizing output feedback model predictive control for constrained nonlinear systems. - IEEE Trans. Automat. Contr., Vol. 49, No. 7, pp. 1172-1177.

Wu N.E., Zhang Y. and Zhou K. (2000): Detection, estimation and accommodation of loss of control effectiveness. — Int. J. Adapt. Contr. and Signal Process., Vol. 14, No. 7, pp. 775-795.

Zhang Y. and Jiang J. (2001): Integrated active fault-tolerant control using IMM approach. - IEEE Trans. Aerospace Electron. Syst., Vol. 37, No. 4, pp. 1221-1235.

Zhang Y. and Jiang J. (2003): Bibliographical review on reconfigurable fault-tolerant control systems. - Proc. IFAC Symp. Safeprocess, Washington, D.C., (CD-ROM).

Zhang Y., Jiang J., Yang Z. and Hussain Z. (2005): Managing performance degradation in fault tolerant control systems. - Proc. 16th IFAC World Congress, Prague, Czech Republic, (CD-ROM). 